\title{
Dynamic capillary pressure analysis of tight sandstone based on digital rock model
}

\author{
Yixin Cao, Mingming Tang ${ }^{\circledR} *$, Qian Zhang, Jiafan Tang, Shuangfang Lu \\ School of Geoscience, China University of Petroleum (East China), Qingdao 266580, P. R. China
}

Keywords:

Dynamic capillary pressure

digital rock model

U-net

lattice Boltzmann method

\section{Cited as:}

Cao, Y., Tang, M., Zhang, Q., Tang, J., $\mathrm{Lu}, \mathrm{S}$. Dynamic capillary pressure analysis of tight sandstone based on digital rock model. Capillarity, 2020, 3(2): 28-35, doi:

10.46690/capi.2020.02.02.

\begin{abstract}
:
In recent studies, dynamic capillary pressure has shown significant impacts on the flow behaviors in porous media under transient flow condition. However, the effect of dynamic capillary pressure effect on tight sandstone is still not very clear. Since lattice Boltzmann method (LBM) is a very promising and widely used method in analyzing flow behaviors, therefore, a two-phase D3Q27 LBM model is adopted in this paper to simulate the flow behaviors and analyze the dynamic capillary pressure effect in tight sandstone. Moreover, a new pore segmentation method for tight sandstone base on U-net deep learning model is implemented in this study to improve the pore boundary qualities of pore space, which is crucial for two-phase LBM simulation of tight sandstone. A total of 3800 3D sub-volume data sets extracted from computed tomography data of 19 tight sandstone samples are selected as ground truth data to train the network and segment the pore space afterward. The simulation results based on the segmented digital rock model, show that nonwetting phase fluid prefer the path with lower dynamic capillary pressure in the seepage process before breaking through the porous model. Furthermore, the increase of injection rate causes the saturation changes more quickly, injection rate also shows apparent positive correlation relationship with capillary pressure, which implies that dynamic capillary pressure effect also exists in tight sandstone, and LBM based two-phase flow simulation could be used to quantitatively analyze such effect in tight sandstone.
\end{abstract}

\section{Introduction}

Capillary pressure plays a vital role in multiphase flow of tight sandstone (Chen et al., 1991; Landry et al., 2014; Tang et al., 2017), it is a generic term that includes dynamic capillary pressure and static capillary pressure (Das et al., 2005; Abidoye et al., 2014; Cai et al., 2014). Recent studies indicate that dynamic capillary pressure is better than static capillary pressure concept for describing transient two-phase flow under dynamic conditions (Hassanizadeh et al., 2002; Dahle et al., 2005; Niasar et al., 2010; Bottero et al., 2011; Diamantopoulos et al., 2016; Li et al., 2018c). To study the mechanism of dynamic capillary pressure, many simulation methods are used to simulate the multiphase flow behaviors in pore-scale, including smooth particle hydrodynamic methods (Tartakovsky and Meakin, 2006; Kunz et al., 2016), fluid volume method (Blunt et al., 2002; Raeini et al., 2014), and lattice Boltzmann method (LBM). Comparing with other numerical methods (Zhu and Li, 2020), LBM can adapt to capillary pressure and viscous force while maintaining the geometry of pore space (Cekmer et al., 2016; Tang et al.,
2018), and it is a widely used and promising method in simulation transient flow behaviors (Wang et al., 2019; Zhang et al., 2019). We implement a new pore segmentation method to optimize the digital rock model of tight sandstone. Then, based on the constructed digital rock model, we carry out twophase flow simulations based on LBM, to analyze the dyanmic capillary pressure effect in tight sandstone.

Recent years, many researches have been carried out on pore segmentation of tight sandstone. From the qualitative evaluation of pores develops into the quantitative evaluation of pore distribution at the micron-nanometer level (Loucks et al., 2009; Andrew et al., 2014; Liu et al., 2018; Zhu et al., 2018; Li et al., 2019; Qu et al., 2020; Shan et al., 2020; Zhao et al., 2020), from 2D analysis relying on scanning electron microscopy (SEM) develops into 3D feature analysis using focused ion beam scanning electron microscopy (FIBSEM) (Curtis et al., 2012; Tartakovsky et al., 2015; Shaina et al., 2016; Alexandra et al., 2020; Chen et al., 2020; Choi et al., 2020; Goral et al., 2020). But most of these researches are focus on imaging technology, the essence of the pore segmentation method is still based on the gray-scale threshold

\footnotetext{
\begin{tabular}{l|l} 
Yandy & ${ }^{*}$ Corresponding author.
\end{tabular} 


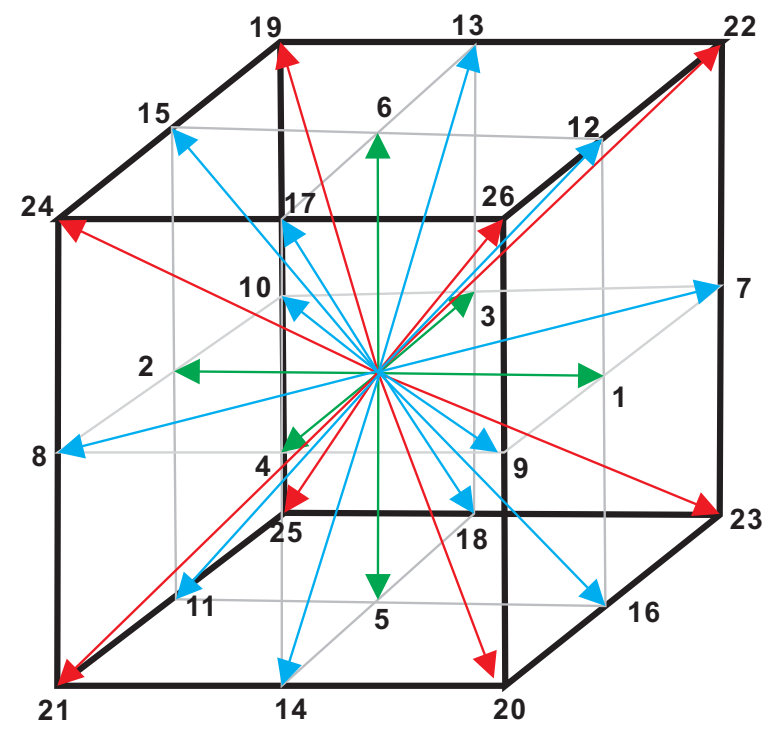

Fig. 1. D3Q27 model schematic diagram.

without any significantly improvement (Kang et al., 2019; Shou et al., 2020). While using the gray-scale threshold to segment the pores from the rock, lots of uncontrollable reasons can cause the inconsistent of gray-scale ranges (Chen et al., 2017). It will cause serious consequences, such as appear a large number of isolated pores which disconnect from each other (Tavanaei and Salehi, 2015), or a few small pores connected by the throat are mistakenly thought to be a large pore (Liu and Ostadhassan, 2017). With lots of iterative training, Unet model can record the 3D representation parameters of all pores (LeCun et al., 2015). What's more, it can also take into account the topological structure of most pore areas (Wang et al., 2020; Xu et al., 2020). Therefore, U-net can segment the pores from the rock more accurately and improve the pore boundary qualities.

In this paper, the pores of digital rock model will be segmented by U-net model. The training data set and the testing data set of U-net are manually modified based on the gray-scale threshold pore segmentation results. Based on the results of pore segmentation, LBM two-phase flow simulation is carried out to study the dynamic capillary pressure mechanism of tight sandstone.

\section{Methods}

\subsection{LBM basic principle}

3D LBM method is used to simulate the rock displacement characteristics of 3D digital rock in this paper. There are four common 3D lattice Boltzmann models: D3Q15, D3Q19, D3Q24 and D3Q27, which discretize the continuous velocity direction of fluid into 15, 19, 24 and 27 velocity components in 3D, respectively (Tang et al., 2018, 2019a, 2019b). In order to improve the calculation accuracy, the D3Q27 model is adopted in this simulation (Fig. 1).

LBM is a method to reconstruct the discrete model of mathematical physics problems, the macroscopic properties of physical systems are calculated by evolutionary equations. In porous media, the pore space is discretized into a series of regular grids (Wang et al., 2019; Zhang et al., 2019). The evolution of fluid particle distribution function on the grid is used to simulate the macroscopic movement law of fluids. The particle distribution function satisfies the following Boltzmann transport equation (Tang et al., 2018, 2019a, 2019b):

$$
f_{k}^{\alpha}\left(x+e_{k} \Delta t, t+\Delta t\right)-f_{k}^{\alpha}(x, t)=\frac{\Delta t\left[f_{k}^{\alpha}(x, t)-f_{k}^{\alpha, e q}(x, t)\right]}{\tau^{\alpha}}
$$

where $k$ is the discrete velocity number; $f_{k}^{\alpha, e q}(x, t)$ is the particle distribution function; $\Delta t$ is the time step length; the "eq" on $f_{k}^{\alpha, e q}(x, t)$ denotes the distribution function at equilibrium; $\alpha$ is a fluid phase; $\tau$ is the relaxation factor based on Boltzmann transport equation, the BGK equation can be obtained as follows:

$$
\frac{\partial f_{k}^{\alpha}}{\partial t}+\boldsymbol{e}_{k} \cdot \nabla f_{k}^{\alpha}=\frac{f_{k}^{\alpha, e q}(x, t)-f_{k}^{\alpha}(x, t)}{\tau^{\alpha}}
$$

The relationship between fluid viscosity $\mu^{\alpha}$, relaxation factor $\tau$, and sound velocity $c_{s}$ can be deduced from the BGK equation:

$$
\mu^{\alpha}=c_{s}^{2}\left(\tau^{\alpha}-\frac{\Delta t}{2}\right)
$$

In order to complete the solution, the equilibrium distribution function $f_{k}^{\alpha, e q}(x, t)$ is given as:

$$
\begin{aligned}
& f_{k}^{\alpha, e q}(x, t)=w_{k} \rho^{\alpha} \\
& \times\left\{1+\frac{\boldsymbol{e}_{k} \cdot \boldsymbol{u}^{\alpha}(x, t)}{c_{s}^{2}}+\frac{\left[\boldsymbol{e}_{k} \cdot \boldsymbol{u}^{\alpha}(x, t)\right]^{2}-c_{s}^{2}\left|\boldsymbol{u}^{\alpha}(x, t)\right|^{2}}{2 c_{s}^{4}}\right\}
\end{aligned}
$$

where $|\cdot|$ is the absolute value symbol, $w_{k}$ is a distribution factor, $\rho^{\alpha}$ is the density of phase $\alpha$, and $\boldsymbol{u}^{\alpha}(x, t)$ is the phase velocity:

$$
\begin{gathered}
\rho^{\alpha}=\Sigma_{k} f_{k}^{\alpha}(x, t) \\
\boldsymbol{u}^{\alpha}=\frac{1}{\rho^{\alpha}} \Sigma_{k} f_{k}^{\alpha} \boldsymbol{e}_{k}
\end{gathered}
$$

Multiphase flow simulation is achieved by Shan-Chen pseudo force model. According to the Shan-Chen model, the pseudo force $F_{f f}^{\alpha}$ acting on phase $\alpha$ (Sukop and Thorne, 2006) can be written as:

$$
F_{f f}^{\alpha}=-\Psi_{\alpha}(x, t) \Sigma_{\bar{\alpha}=1}^{S} \Sigma_{k=0}^{b} G_{\alpha \bar{\alpha}} w_{k} \Psi_{\bar{\alpha}}\left(x+e_{k} \Delta t, t\right) \boldsymbol{e}_{k}
$$

where $\Psi_{\alpha}(x, t)$ is constraint force function, $S$ is phase code. Shan-Chen model updates the velocity of each phase through the equilibrium velocity $\boldsymbol{u}(x, t)^{\prime}$, which is defined as:

$$
\boldsymbol{u}(x, t)^{\prime}=\frac{\sum_{\alpha=1}^{S} \Sigma_{k=1}^{b} \frac{f_{k}^{\alpha} e_{k}}{\tau^{\alpha}}}{\sum_{\alpha=1}^{S} \Sigma_{k=1}^{b} \frac{f_{k}^{\alpha}}{\tau^{\alpha}}}
$$

After the completion of LBM simulation, we propose a model for the calculation of dynamic capillary pressure based 


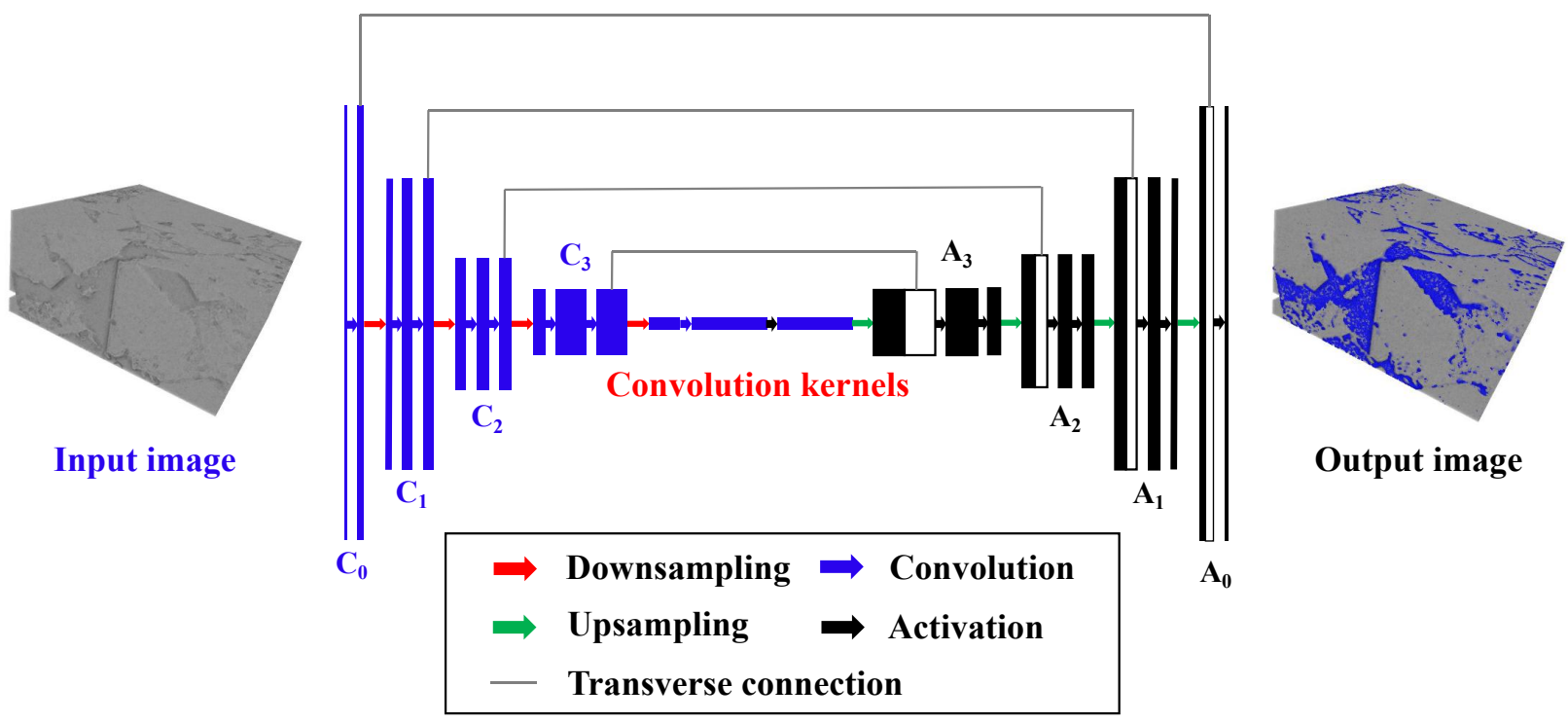

Fig. 2. Schematic diagram of U-net based on segmentation method.

on the law of conservation of energy (Tang et al., 2017, 2018, 2019a, 2019b; Li et al., 2018a, 2018b), which achieves good results in conventional porous media, and its expression is written as:

$$
P_{D C}=\frac{d W_{p}^{e x t}-d W_{v i s}-d E_{k i n}}{Q d t}, \quad(Q \neq 0)
$$

where $P_{D C}$ is dynamic capillary pressure, $W_{p}^{e x t}$ is the work of external pressure, $W_{v i s}$ is the work of viscous force, $E_{k i n}$ is the kinetic energy, and $Q$ is the flow rate:

$$
Q=\frac{d V}{d t}
$$

where $V$ is the volume of injected fluid. The essence of Eq. (9) is conservation of energy. The work done by external pressure provides input energy $\left(W_{p}^{e x t}\right)$, which is consumed by viscous resistance $\left(W_{v i s}\right)$ and dynamic capillary pressure $\left(P_{D C}\right)$, the rest is converted into kinetic energy $\left(E_{k i n}\right)$. It is worth noting that in the stationary state $(Q=0)$ this formula does not apply.

\subsection{Pore segmentation based on U-net}

U-net uses jump join to fuse multi-scale low-level finegrained information and high-level abstract semantic information to achieve accurate segmentation. By encoding and decoding the image features and fusing the semantic features of the high and low layers of the network, a better segmentation effect can be achieved (Xu et al., 2018; Xiang et al., 2019; Zhang et al., 2019). Comparing with other deep learning networks, U-net can complete model training and image segment with a small sample size. In the process of U-net iterative training, in order to maximize the use of GPU memory, we tend to reduce the number of input batches and increase the number of input images in each batch (Ronneberger et al., 2015).

\section{Data preparation}

Fig. 2 shows the U-net structure used in this paper. In Fig. 2, the blue blocks represent the convolutional layer and the black blocks represent the transposed-convolutional layer. The gray line represents replication, the red arrow represents convolution, and the green arrow represents activation (transposed-convolution). The inputs and outputs of the network are all images. The size of the input layer $\mathrm{C}_{0}$ is $240 \times 240 \times 50$. Layer $C_{0}$ convolve to layer $C_{1}, C_{1}$ 's size is $120 \times 120 \times 100$. After three times of convolution to the convolution kernel, the activation function is used to activate it. Layer $\mathrm{A}_{3}$ 's size is $15 \times 15 \times 400$, and layer $\mathrm{A}_{2}$ 's size is $30 \times 30 \times 200$. A total of three times of activation to the image output layer $\mathrm{A}_{0}$. In the training process of the network, the gray-scale change law of the region matching the pores is stored in the form of convolution kernel, which can be used for pore extraction of other images. What's more, U-net can analyze the spatial topology around the pores through a series of the convolution rocks from small to large, which is very important for the correct extraction of pores.

The training set and testing set of U-net model are very important, they directly affect the training result of U-net. Therefore, in the process of preparing the training set and testing set, we use manually modify the gray-scale threshold segmentation result, and the final processing result is shown in Fig. 3. Before we start training, it is necessary to expand the image data. A total of 3800 3D data sets extracted from 19 tight sandstone samples are selected as basic data to train the network, and data enhancement is carried out before the training process by random cut and rotation. In the testing process, 185 images (150 training pieces, 35 testing pieces) and 185 labels (150 training pieces, 35 testing pieces) are randomly selected for each batch. After the training, the segmentation results are presented in the form of 3D.

In order to analyze the 3D flow behaviors and improve the 


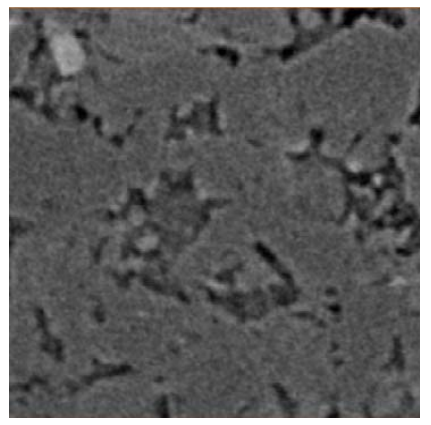

(a)

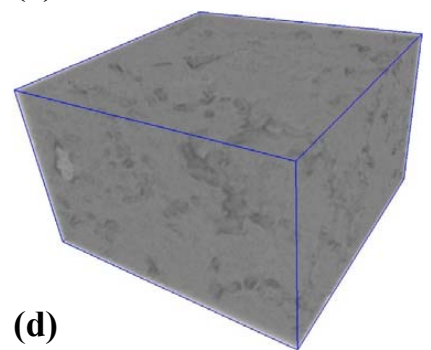

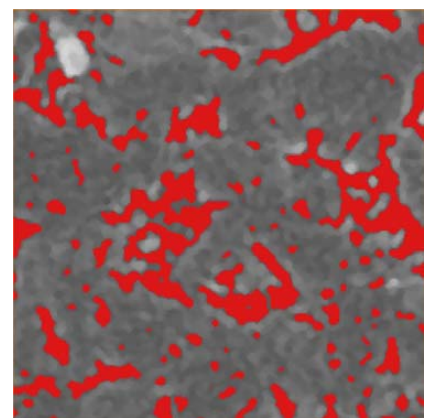

(b)

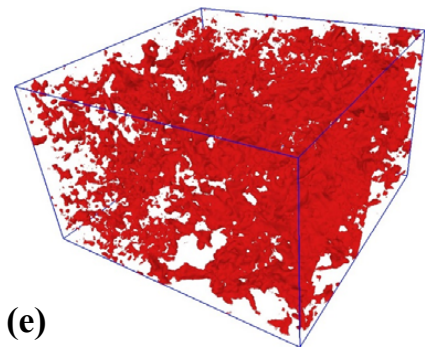

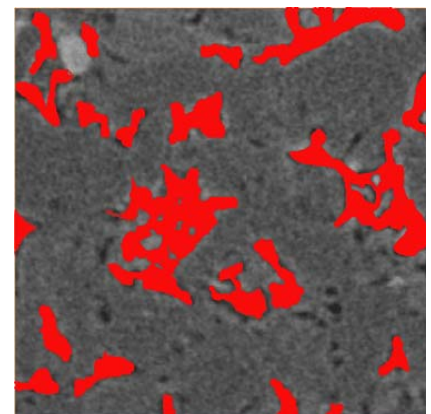

(c)

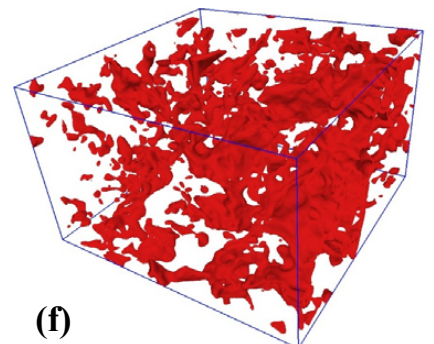

Fig. 3. Schematic diagram of training samples ((a) shows $2 \mathrm{D}$ scan-image of the rock, (b) shows $2 \mathrm{D}$ pore segmentation results by gray-scale threshold, (c) shows 2D image manual calibration result, (d) shows 3D scan-image of the rock, (e) shows 3D pore segmentation results by gray-scale threshold, and (f) shows 3D image manual calibration result).

Table 1. D3Q27 model system parameters.

\begin{tabular}{llll}
\hline Model & $c_{s}$ & Parameters & $W_{k}$ \\
\hline & & $(0,0,0)$ & $8 / 27(k: 0)$ \\
D3Q27 & $\frac{1}{\sqrt{3}}$ & $( \pm 1,0,0),(0, \pm 1,0),(0,0, \pm 1)$ & $2 / 27(k: 1-6)$ \\
& & $( \pm 1, \pm 1,0),( \pm 1,0, \pm 1),(0, \pm 1, \pm 1)$ & $1 / 54(k: 7-18)$ \\
& & $( \pm 1, \pm 1, \pm 1)$ & $1 / 216(k: 19-26)$ \\
\hline
\end{tabular}

calculation accuracy, we chose D3Q27 model with the highest accuracy for simulation. The parameters of the model system are shown in Table 1.

\section{Results}

\subsection{Pore segmentation based on U-net}

After iterative training of U-net, the final pore segmentation results are shown in Fig. 4. The size of the generated model is $240 \times 240 \times 50$, and the number of training epochs is 500 . As shown in Figs. 4(b2), 4(c2), and 4(d2), as the with training epochs progress, U-net can identify pores more and more accurately, isolated pores are gradually eliminated, and the segmentation results are gradually improved.

In addition, as shown in Figs. 4(d2) and 4(e2), the segmentation effect of U-net is obviously better than the traditional gray-scale threshold segmentation, the boundary of pores are also smoother. The traditional gray-scale threshold segmentation method can only set a specific threshold simply. Due to the influence of many factors, the gray-scale scale range of the rock is not consistent. There will be many isolated pores that are not connected to each other in the segmentation result, as shown in Fig. 5(e2), but these pores do not exist in reality. With iterative training, U-net can take into account the pore grayscale threshold range and the pore 3D topology, which has obvious advantages comparing with the traditional threshold division method, as shown in Fig. 5(d2).

\subsection{Dynamic capillary pressure simulation based on $L B M$}

Fig. 5 shows the simulation results of the nonwetting phase flow process in a reconstructe porous model generated from Unet based segmentation. The displacing process with a uniform wettability of $140^{\circ}$ and different injection rate of $0.3 \mathrm{~mm} / \mathrm{s}$ and $0.5 \mathrm{~mm} / \mathrm{s}$. When the nonwetting phase invades the porous model that is initially occupied by the wetting phase, a convex nonwetting phase front is formed as a result of the hydrophilic characteristic of sandstone (Figs. 5(a1) and 5(b1)). When more nonwetting phase fluid is injected into the sandstone, the nonwetting phase fluid selects preferential paths with lower capillary resistance (Fig. 5(a2) and 5(b2)). When further nonwetting phase fluid is injected into the sandstone, the nonwetting phase fluid finally breaks through the porous model (Fig. 5(a3) and 5(b3)). Raeini et al. (2014) also presented a similar nonwetting phase flow process in a more hydrophilic sandstone based on the pseudo-potential LBM. Unfortunately, 
(a1)

(b1)

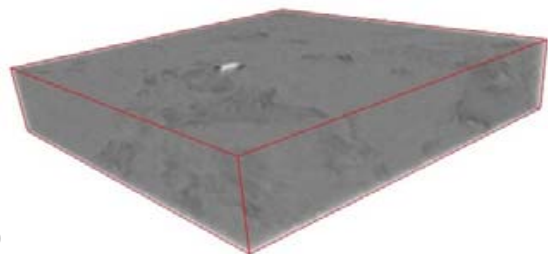

(c1)

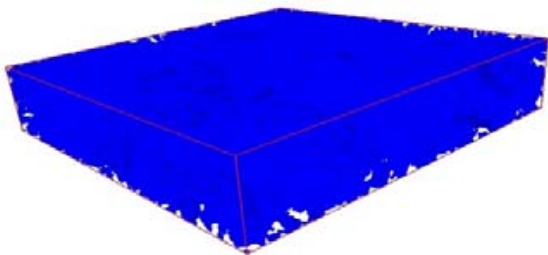

(d1)

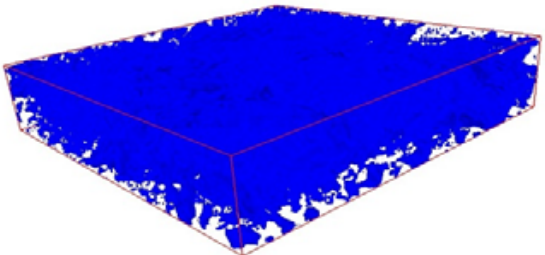

(e1)
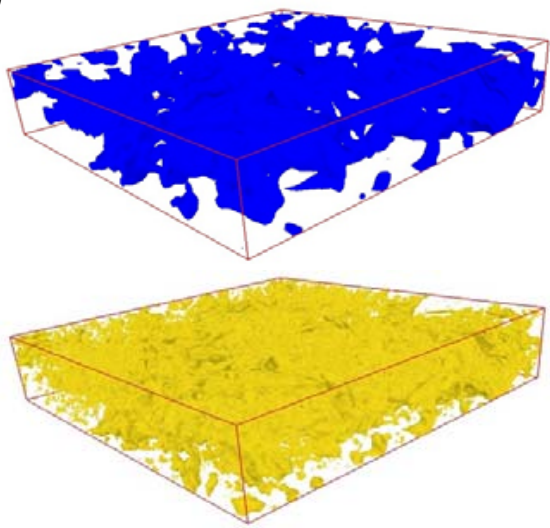

(a2)

(b2)
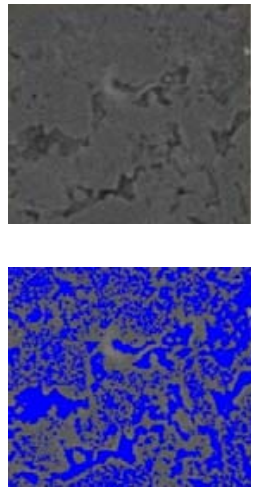

(c2)

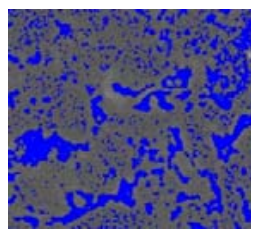

(d2)

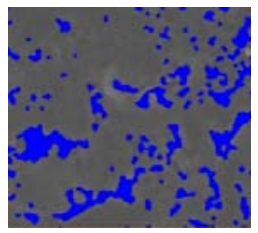

(e2)

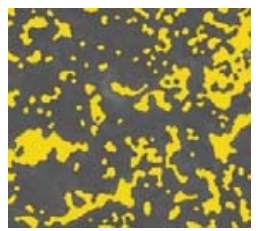

Fig. 4. U-net pore segmentation result diagram, the left column represents the $3 \mathrm{D}$ image, the right column represents the 2D image, where (a1) and (a2) show scan-image of the rock, (b1) and (b2) show pore segmentation training result on epoch 10, (c1) and (c2) show pore segmentation training result on epoch 100, (d1) and (d2) show pore segmentation final results, (e1) and (e2) show traditional gray-scale threshold pore segmentation result.

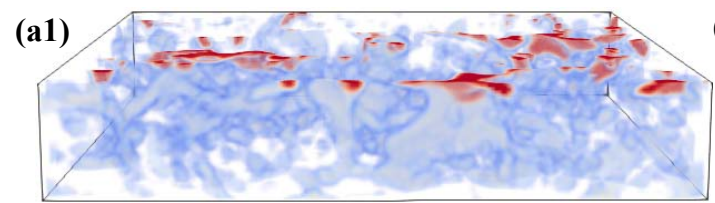

(a2)

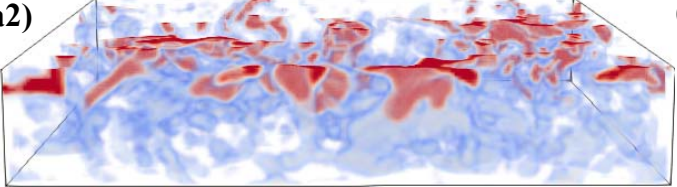

(a3)

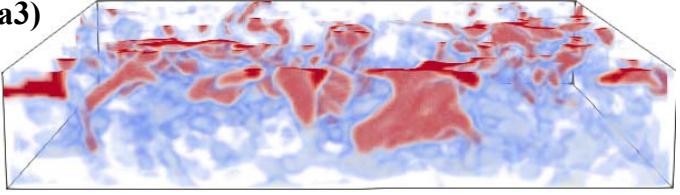

Case A

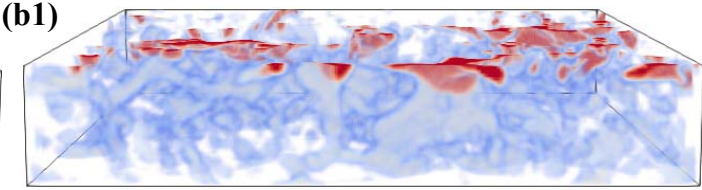

(b2)

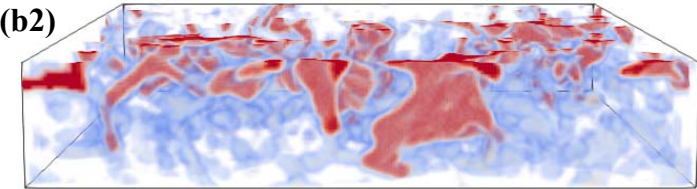

(b3)

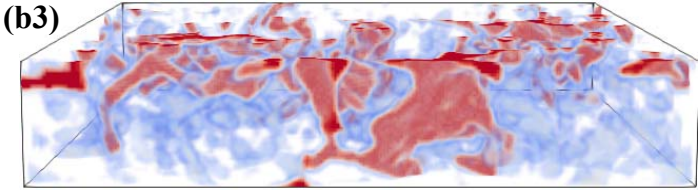

Case B

Fig. 5. Snapshots of crude oil behavior during oil injection with a contact angle of $140^{\circ}$ and different injection speeds. The left column represents the simulation results of case A with an injection speed of $0.3 \mathrm{~mm} / \mathrm{s}$, while the right column represents the simulation results of case B with injection speed of $0.5 \mathrm{~mm} / \mathrm{s}$, where (a1), (a2) and (a3) show the snapshots of 40, 60, $80 \mathrm{~ms}$, respectively, and (b1), (b2) and (b3) show the snapshots of 30 , 45 and 55 ms, respectively. The red color represents the displacing nonwetting phase; the light blue color represents the pore space filled with the wetting phase. The fluids are set a partially transparent, while the solid matrix is set as completely transparent to highlight the distribution of fluids. 


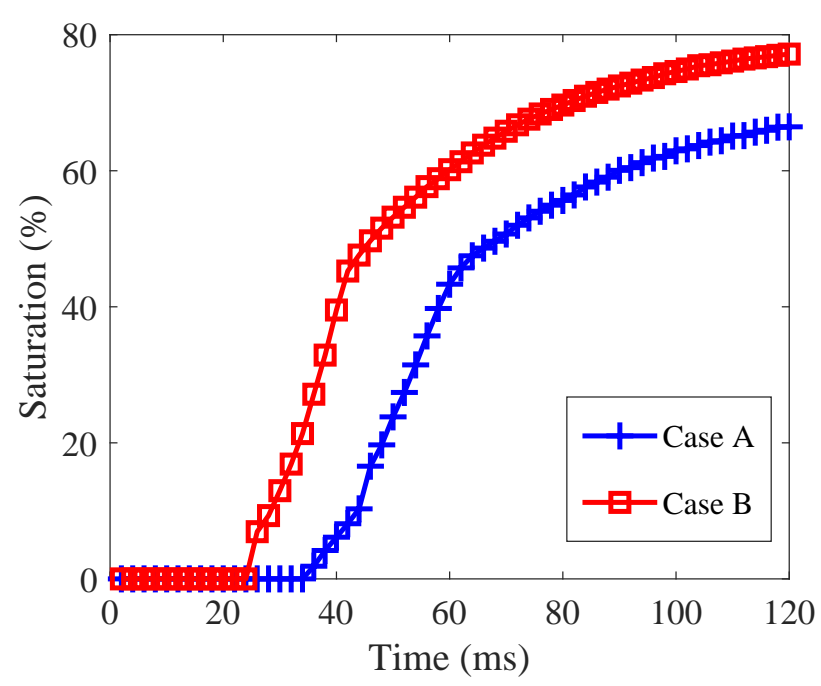

(a)

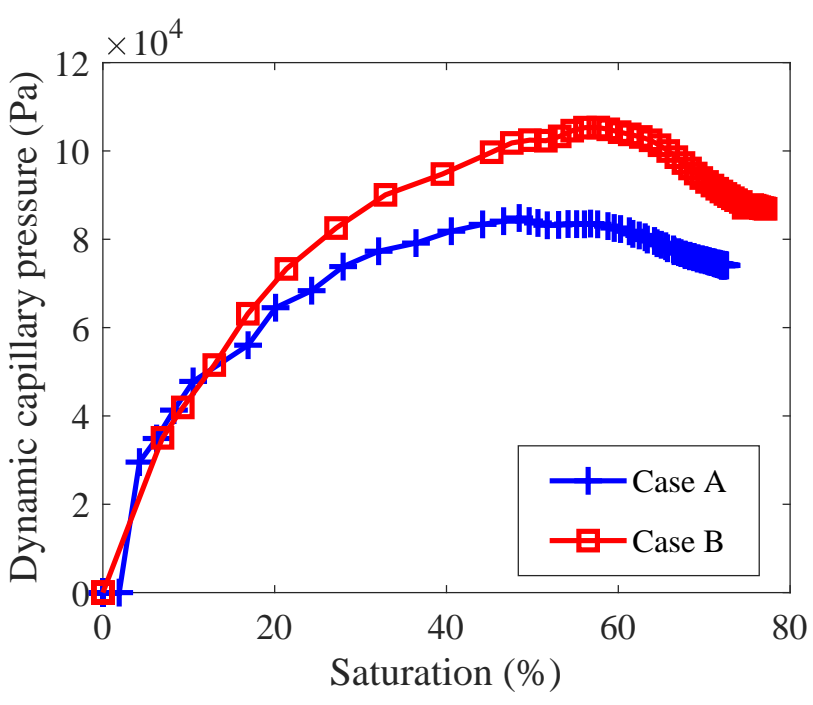

(b)

Fig. 6. Evolution of the saturation versus time and the dynamic capillary pressure versus saturation curves. (a) Saturation versus time of case A and B with different injection speeds. (b) dynamic capillary pressure-saturation curves for case A with injection speed of $0.3 \mathrm{~mm} / \mathrm{s}$ and case B with injection speed of $0.5 \mathrm{~mm} / \mathrm{s}$.

dynamic capillary pressure and the breakthrough process are not considered in their simulations.

Fig. 6(a) shows the results of saturation as a function of time for cases A and B. Because the saturation of the nonwetting phase $\left(S_{n w}\right)$ and the saturation of the wetting-phase $\left(S_{w}\right)$ is conjugated as:

$$
S_{n w}=1-S_{w}
$$

We only discuss the result of the nonwetting phase saturation in the following. It is worth noting that the evolution curves of saturation fluctuate when the nonwetting phase starts to entry the porous media, due to the boundary effects, then the saturation will increase almost linearly before breakthrough time. Fig. 6(a) shows that a higher injection rate leads to a more rapid change of the saturation, and the nonwetting phase saturation ultimately reaches an asymptotic limit.

Fig. 6(b) shows curves of dynamic capillary pressure versus the wetting phase saturation of cases $A$ and B. It shows that the dynamic capillary pressure is larger for a larger injection rate. Fig. 6(b) also shows non-monotonic trend for all cases, such phenomenon is also called overshoot, which is agree with the experimental results of Bottero et al. (2011), while studying dynamic capillary pressure with a constantpressure boundary condition.

\section{Conclusions}

A U-net based pore segmentation method is established to optimize 3D digital rock model. Then, different LBM twophase displacing simulations with different injection speeds are used to analyze the dynamic capillary pressure effect in tight sandstone. The results show that the U-net can optimize the digital rock model by taking into account both the gray threshold and topological structure of pore structure. Dynamic capillary pressure effect is recovered based on digital rock model and LBM method. The simulation results show that the injection speed affect the absolute value of dynamic capillary pressure. The higher the injection speed, the greater the capillary pressure, and a non-monotonic trend of dynamic capillary pressure are also recovered from the proposed U-net based LBM method. It shows that the proposed method can effectively analyze dynamic effects, and further study about the U-net based minerals segmentation and dynamic capillary pressure simulation will be carried out in the very near future.

\section{Acknowledgement}

This study is supported by the Foundation of Shandong Province (Nos. ZR2019MD006, ZR2014DL003), the Natural Science Foundation of China (No. 41402108) and Talent Introduction Funding (No. 2013059), China University of Petroleum (East China) Financing Innovation Funding (Nos. 2014010336 and 27R1510046A). We would like to thank the China Scholarship Council for their financial support. The authors want to express their sincere appreciation of the constructive comments made by the three anonymous reviewers and editor for reviewing the manuscript.

\section{Conflict of interest}

The authors declare no competing interest.

Open Access This article is distributed under the terms and conditions of the Creative Commons Attribution (CC BY-NC-ND) license, which permits unrestricted use, distribution, and reproduction in any medium, provided the original work is properly cited.

\section{References}

Abidoye, L.K., Das, D.B. Scale dependent dynamic capillary pressure effect for two-phase flow in porous media. Adv. Water Resour. 2014, 74: 212-230. 
Alexandra, R., Dubravka, P., Wu, K., et al. 3D pore system reconstruction using nano-scale 2D SEM images and pore size distribution analysis for intermediate rank coal matrix. Fuel 2020, 275: 117934.

Andrew, M., Bijeljic, B., Blunt, M.J. New frontiers in experimental geoscience: X-ray microcomputed tomography and fluid flow. Microsc. Anal. 2014, 28(2): 4-7.

Blunt, M.J., Jackson, M.D., Piri, M., et al. Detailed physics, predictive capabilities and macroscopic consequences for pore-network models of multiphase flow. Adv. Water Resour. 2002, 25(8-12): 1069-1089.

Bottero, S., Hassanizadeh, S.M., Kleingeld, P.J., et al. Nonequilibrium capillarity effects in two-phase flow through porous media at different scales. Water Resour. Res. 2011, 47(10): W10505.

Cai, J., Perfect, E., Cheng, C.L., et al. Generalized modeling of spontaneous imbibition based on Hagen-Poiseuille flow in tortuous capillaries with variably shaped apertures. Langmuir 2014, 30(18): 5142-5151.

Cekmer, O., Um, S., Mench, M.M. A combined pathpercolation-Lattice-Boltzmann model applied to multiphase mass transfer in porous media. Int. J. Heat Mass Transf. 2016, 93: 257-272.

Chen, C., Lu, S., Li, J., et al. Digital core modeling construction of different lithofacies shale: A case study of dongying depression. Geoscience 2017, 31(5): 10691078. (in Chinese)

Chen, S., Chen, H., Martnez, D., et al. Lattice Boltzmann model for simulation of magnetohydrodynamics. Phys. Rev. Lett. 1991, 67(27): 3776-3779.

Chen, X., Qu, X., Xu, S., et al. Dissolution pores in shale and their influence on reservoir quality in Damintun Depression, Bohai Bay Basin, East China: Insights from SEM images, $\mathrm{N}_{2}$ adsorption and fluid-rock interaction experiments. Mar. Pet. Geol. 2020, 117: 104394.

Choi, C., Lee, Y., Song, J., et al. Equivalent pore channel model for fluid flow in rock based on microscale X-ray CT imaging. Materials 2020, 13(11): 2619.

Curtis, M.E., Sondergeld, C.H., Ambrose, R.J., et al. Microstructural investigation of gas shales in 2D and 3D using nano-meterscale resolution imaging. AAPG Bull. 2012, 96(4): 665-677.

Dahle, H.K., Celia, M.A., Hassanizadeh, S.M. Bundle-of-tubes model for calculating dynamic effects in the capillarypressure-saturation relationship. Transp. Porous Media 2005, 58(1-2): 5-22.

Das, D.B., Hanspal, N.S., Nassehi, V. Analysis of hydrodynamic conditions in adjacent free and heterogeneous porous flow domains. Hydrol. Process. 2005, 19(14): 2775-2799.

Diamantopoulos, E., Durner, W., Harter, T. Prediction of capillary air-liquid interfacial area vs. saturation function from relationship between capillary pressure and water saturation. Adv. Water Resour. 2016, 97: 219-223.

Goral, J., Andrew, M., Olson, T., et al. Correlative core-to pore-scale imaging of shales. Mar. Pet. Geol. 2020, 111: 886-904.
Hassanizadeh, S.M., Celia, M.A., Dahle, H.K. Dynamic effects in the capillary pressure saturation relationship and their impacts on unsaturated flow. Vadose Zone J. 2002, 1(1): 38-57.

Kang, D., Yang, E., Yun, T. Stokes-Brinkman flow simulation based on 3-D $\mu$-CT images of porous rock using grayscale pore voxel permeability. Water Resour. Res. 2019, 55(5): 4448-4464.

Kunz, P., Zarikos, I.M., Karadimitriou, N.K., et al. Study of multi-phase flow in porous media: Comparison of SPH simulation with micro-model experiments. Transp. Porous Media 2016, 114(2): 581-600.

Landry, C.J., Karpyn, Z.T., Ayala, O. Relative permeability of homogenous-wet and mixed-wet porous media as determined by pore-scale lattice Boltzmann modeling. Water Resour. Res. 2014, 50(5): 3672-3689.

LeCun, Y., Bengio, Y., Hinton, G. Deep learning. Nature 2015, 521(7553): 436-444.

Li, J., Ho, M.T., Wu, L., et al. On the unintentional rarefaction effect in LBM modeling of intrinsic permeability. Adv. Geo-Energy Res. 2018a, 2(4): 404-409.

Li, J., Wang, Z., Wei, J., et al. The numerical simulation of the shale gas fluid-structure interaction based on the digital rock and LBM. Scientia Sinica (Technologica) 2018b, 48(5): 499-509. (in Chinese)

Li, M., Guo, Y., Li, Z., et al. Pore-throat combination types and gas-water relative permeability responses of tight gas sandstone reservoirs in the Zizhou area of east Ordos Basin, China. Acta Geol. Sin.-Engl. Ed. 2019, 93(3): 622-636.

Liu, H., Yang, Y., Wang, F., et al. Micro pore and throat characteristics and origin of tight sandstone reservoirs: A case study of the Triassic Chang 6 and Chang 8 members in Longdong area, Ordos Basin, NW China. Pet. Explor. Dev. 2018, 45(2): 239-250.

Liu, K., Ostadhassan, M. Multi-scale fractal analysis of pores in shale rocks. J. Appl. Geophys. 2017, 140: 1-10.

Li, Y., Li, H., Cai, J., et al. The dynamic effect in capillary pressure during the displacement process in ultra-low permeability sandstone reservoirs. Capillarity 2018c, 1(2): 11-18.

Loucks, R.G., Reed, R.M., Ruppel, S.C., et al. Morphology, genesis, and distribution of nanometer-scale pores in siliceous mudstone of the Mississippian Barnett shale. J. Sediment. Res. 2009, 79(12): 848-861.

Niasar, V.J., Hassanizadeh, S.M., Dahle, H.K. Non-equilibrium effects in capillarity and interfacial area in two-phase flow: Dynamic pore-network modelling. J. Fluid Mech. 2010, 655: 38-71.

Qu, Y., Sun, W., Tao, R., et al. Pore-throat structure and fractal characteristics of tight sandstones in Yanchang Formation, Ordos Basin. Mar. Pet. Geol. 2020, 120: 104573.

Raeini, A.Q., Bijeljic, B., Blunt, M.J. Numerical modelling of sub-pore scale events in two-phase flow through porous media. Transp. Porous Media 2014, 101(2): 191-213.

Ronneberger, O., Fischer, P., Brox, T. U-net: Convolutional networks for biomedical image segmentation. Paper 
Presented at 18th International Conference on Medical Image Computing and Computer-Assisted Intervention (MICCAI), Munich, Germary, 5-9 October, 2015.

Shaina, K., Hesham, E., Carlos, T., et al. Assessing the utility of FIB-SEM images for shale digital rock physics. Adv. Water Resour. 2016, 95: 302-316.

Shan, C., Zhao, W., Wang, F., et al. Nanoscale pore structure heterogeneity and its quantitative characterization in Chang7 lacustrine shale of the southeastern Ordos Basin, China. J. Pet. Sci. Eng. 2020, 187: 106754.

Shou, Y., Zhao, Z., Zhou, X. Sensitivity analysis of segmentation techniques and voxel resolution on rock physical properties by X-ray imaging. J. Struct. Geol. 2020, 133: 103978.

Sukop, M.C., Thorne, D.T. Lattice Boltzmann Modeling: An Introduction for Geoscientists and Engineers. New York, Springer, 2006.

Tang, M., Lu, S., Zhan, H., et al. The effects of a microscale fracture on dynamic capillary pressure of two-phase flow in porous media. Adv. Water Resour. 2018, 113: 272-284.

Tang, M., Zhan, H., Lu, S., et al. Pore-scale $\mathrm{CO}_{2}$ displacement simulation based on the three fluid phase lattice Boltzmann method. Energy Fuels 2019a, 33: 1003910055.

Tang, M., Zhan, H., Ma, H., et al. Upscaling of dynamic capillary pressure of two-phase flow in sandstone. Water Resour. Res. 2019b, 55(4): 426-443.

Tang, M., Zhao, H., Ma, H., et al. Study on $\mathrm{CO}_{2}$ huff-n-puff of horizontal wells in continental tight oil reservoirs. Fuel 2017, 188: 140-154.

Tartakovsky, A.M., Meakin, P. Pore scale modeling of immiscible and miscible flows using smoothed particle hydrodynamics. Adv. Water Resour. 2006, 29: 1464-1478.

Tavanaei, A., Salehi, S. Pore, throat, and grain detection for rock sem images using digitalwatershed image segmentation algorithm. J. Porous Media 2015, 18(5): 507-518.

Wang, H., Yuan, X., Liang, H., et al. A brief review of the phase-field-based lattice Boltzmann method for multiphase flows. Capillarity 2019, 2(3): 33-52.

Wang, P., Qiang, Y., Yang, X., et al. Double attention 3D-UNet for lung nodule segmentation. Computer Engineering 2020, 2020: 1-10. (in Chinese)

Xiang, Y., Zhao, Y., Dong, J. Remote sensing image mining area change detection based on improved UNet siamese network. Journal of China Coal Society 2019, 44(12): 3773-3780. (in Chinese)

$\mathrm{Xu}, \mathrm{D} ., \mathrm{Li}, \mathrm{H} .$, Zhou, L., et al. Model of automatic identification of diabetic macular edema via convolutional neural networks UNet. Recent Advances in Ophthalmology 2020, 40(4): 357-361. (in Chinese)

Xu, J., Jin, G., Zhu, T. Segmentation of rock images based on U-net. Industrial Control Computer 2018, 31(4): 98-99. (in Chinese)

Zhang, G., Zhang, Y., Xu, A., et al. Microflow effects on the hydraulic aperture of single rough fractures. Adv. GeoEnergy Res. 2019, 3(1): 104-114.

Zhao, J., Wang, P., Zhang, Y. Influence of $\mathrm{CO}_{2}$ injection on the pore size distribution and petrophysical properties of tight sandstone cores using nuclear magnetic resonance. Energy Sci. Eng. 2020, 8(7): 2286-2296.

Zhu, G., Li, A. Interfacial dynamics with soluble surfactants: A phase-field two-phase flow model with variable densities. Adv. Geo-Energy Res. 2020, 4(1): 86-98.

Zhu, R., Jin, X., Wang, X., et al. Multi-scale digital rock evaluation on complex reservior. Earth Science 2018, 43(5): 1773-1782. (in Chinese) 